\title{
Safe Practices for Routine (Non-COVID19 testing) Medical Laboratory
}

\author{
Prashant Goyal*
}

Director \& Chief Pathologist, Accuprobe Healthcare \& Diagnostics Pvt Ltd. Delhi. India

Protection of you and your staff from COVID19 infection will depend on emphasizing basic infection prevention measures, not only in workplace (laboratory) but in their home. All staff should implement good hygiene and infection control practices as recommended by WHO and as guidelines of Central and State Govt. Simple precautions and planning can make a big difference. Main aims of safe practices in laboratories is to stop the transmission chain of COVID19 between patients and staff as well as elimination of the anxiety of patient regarding their safety.

Standard Operating Procedures (SOPs) for sample collection, transportation, processing and disposable, should be prepared according to your setup as well as current National and WHO guidelines, and updated regularly.

Following extra safe practices should be followed along with routine good lab practices.

\section{General Instructions For staff}

\section{(Technical or Non-technical):}

- Man-power should be according to workload (neither overloaded staff nor extra staff). Establishing alternating days or extra shifts (If suitable) that reduce the total number of staff in a lab at a given time, allowing them to maintain the social distance.

- Can be given leave to staff who are elderly, or have history of chronic illness, like heart disease, diabetes, chronic lung disease, immunocompromised etc. Can be posted them at low risk area with extra safety.

- Record of staff's attendance should be maintained by one person. If biometric, staff should be use hand-rub always after thumb impression on biometric.

- Actively screen every staff for fever and symptoms of COVID-19 before they enter the lab.

- All staff should wear masks, gloves, Lab coat, eye protector, shoe cover and maintain social distancing at all time in lab.

- Promote frequent and thorough hand washing with soap and running water or use alcohol-based (containing at least $60 \%$ alcohol) hand rubs by staff.
- Frequently use of hand-rub by staff after touch of common things like desk, door of toilet, refrigerator etc

- Encourage respiratory etiquette, including covering mouth during coughs and sneezes.

- Discourage staff from using other staff' phones, desks, equipment and other work tools, when possible.

- Minimizing contact among staff, by replacing direct face-to-face meetings with virtual communications, if required.

- Provide adequate, appropriate training, and informational material to staff about safety, including proper hygiene practices and the use of any workplace controls.

- Training of staff who need to use protecting clothing and equipment (PPE) how to put it on, use/wear it, and take it off correctly.

- Activities such as eating, drinking, etc to be avoided in lab premises strictly.

- Remove all jewelleries, wrist watch etc which could tear glove material, easily become contaminated or act as a fomite for infection.

- Beard prevents proper fitting of face masks, so regular shaving should be done.

- Avoid excessive use of mobile. Decontaminated mobile and laptop with sprit, time to time and before leaving the laboratory.

- Avoid transport of your belongings like mobile charger, pen etc from lab to home or vice versa.

- Avoid contact of hands (with or without gloves) with the face.

- The floor, work surfaces (e.g. desks, chair door etc) and objects (e.g. telephones, keyboards, printer, etc) should be disinfected twice daily by mopping with a cloth soaked in 1\% sodium hypochlorite (or $70 \%$ alcohol for metallic surfaces).

- Encourage your staff to self-monitor for signs and symptoms of COVID-19. Inform you. if they suspected. 
- $\quad$ Encourage your staff to stay home if he/she is sick (or even his/her close family member is sick).

- Reusable PPE must be cleaned and disinfected according to manufacturer's reprocessing instructions prior to re-use.

- Encourage the staff to wash their clothes daily and wear fresh cloth daily.

- $\quad$ Take bath with warm water and soak all clothes in soap water after reach to home.

- $\quad$ Put sanitizing hand rub dispensers in prominent places around the workplace. Make sure these dispensers are regularly refilled.

- Ask the staff and people to Practise Yoga with Pranayam daily to increase immunity.

\section{Instructions for Patients, Attendant and visitors}

- Allowed only one patient at a time in lab and reduced stay of patient in lab.

- $\quad$ Restrict the number of attendants with patient entering lab. Only one attendant should be allowed to entre in lab, if required.

- $\quad$ Provide and encourage the use of alcohol-based hand rub to all visitors including patient before entering in lab.

- Ensure that patients or visitors should be wearing proper mask, covering nose and mouth before entry to the lab. If not, they should be offered a face-mask.

- $\quad$ Place a signage or poster at entrances indicating your policy for visitors/patients and to provide instructions about hand hygiene and respiratory hygiene.

- Check temperature of patients by infra-red thermal gun, at time of entry.

- In case of Suspected COVID symptoms like Highgrade Fever, Breathlessness, kindly don't take samples and refer them to the Central helpline.

- Avoid un-necessary stay of patients in lab and prefer email or Whatsapp for delivery of the reports to reduce second visit.

- Inside the lab, patients are not allowed to sit anywhere or touch anything.

- Avoid transaction by cash or by card. Preferable method is e-wallets like Paytm, Phone-Pe etc
- Better to avoid patients without doctor's prescription.

- Avoid un-necessary visitors or meetings and use virtual communications or tele-conference, if required.

\section{Instructions for Sample collection,} transportation and processing:

- Home collections to be avoided, if not urgent or if the patient is really sick

- $\quad$ Asked patients to bring urine, semen, sputum samples from home.

- Sample collection room should be near to entry door or outside the laboratory, if possible.

- You can install a transparent plastic shield or curtain between patient's chair and phlebotomist.

- Dedicated staff for sample collection.

- Use disposable Tourniquets (preferably) or sanitize after every use. Rubber tourniquets are easy to sanitize.

- Clean and disinfect environmental surfaces (patient's chair, wall of room, plastic apron, etc) in accordance with protocols after each sample collection.

- N95 mask (or triple layers medical mask), face shield, gloves, full lab coat along with plastic apron should be wear by phlebotomist during sample (non-respiratory) collection.

- Ensure proper ventilation in collection room but avoid use of air conditioners.

- Samples should be packed in leak-proof specimen bags before transportation. Requisition forms should be sent in a separate zip lock pack to avoid crosscontamination.

- All technical procedures should be performed in a way that minimizes the formation of aerosols and droplets.

- After centrifuge, let the sample tubes stand for at least 10 minutes so that the droplets settle down.

- Microbiology work should be performed in a certified BSL-II.

- All the residual samples should be discarded in appropriate disinfectants with confirmed virucide activity against enveloped viruses.

- $\quad$ Double layered biomedical waste bags (using 2 bags) should be used to ensure adequate strength and prevent leakages.

- Biomedical waste rules (latest) should be strictly followed as always. 
Recommended rational use of PPE in routine laboratory

\begin{tabular}{|l|l|l|}
\hline Risk & Staff & Recommended PPE \\
\hline High Risk & $\begin{array}{l}\text { Who collects respiratory samples (Nasal or } \\
\text { oral swab) or aspirates FNA from head \& neck } \\
\text { regions }\end{array}$ & Full PPE \\
\hline Moderate Risk & $\begin{array}{l}\text { Front office/ Receptionist, Phlebotomists, } \\
\text { Technician working in microbiology and any staff } \\
\text { who deal with patients and respiratory samples }\end{array}$ & $\begin{array}{l}\text { N95 mask or equivalent, Eye protection, Double } \\
\text { layer of Gloves, Lab coat with plastic apron and other } \\
\text { universal precautions as in routine. (Full PPE is better, } \\
\text { if available) }\end{array}$ \\
\hline Low risk & $\begin{array}{l}\text { Admin, technician at haematology, biochemistry, } \\
\text { serology (anyone who does not deal with } \\
\text { patients and respiratory samples) }\end{array}$ & $\begin{array}{l}\text { Surgical mask, Gloves, Lab coat and other universal } \\
\text { precautions as in routine. (Full PPE is not required) }\end{array}$ \\
\hline
\end{tabular}

Last but not the least, any incident involving potential or actual exposure to novel COVID-19 should be immediately reported to authority and any affected area/equipment decontaminated as recommended.

\section{References}

1. Guidance on Preparing Workplaces for COVID-19, 2020 available at https://www.osha.gov/Publications/OSHA3990. pdf
2. Interim Laboratory Biosafety Guidelines for Handling and Processing Specimens Associated with Coronavirus Disease 2019 (COVID-19) available at https://www.cdc.gov/ coronavirus/2019-nCoV

3. Guidelines by Indian Council of Medical Research (ICMR), New Delhi, available at https://main.icmr.nic.in/content/ covid-19

4. Guidelines by World Health Organization, available at https:// www.who.int/emergencies/diseases/novel-coronavirus-2019

*Corresponding author:

Dr. Prashant Goyal, Director \& Chief Pathologist, Accuprobe Healthcare \& Diagnostics Pvt Ltd., B14/7, Jhilmil Industrial Area, Delhi-110095, India Email: prashant@accuprobe.in

Financial or other Competing Interests: None. 